\title{
Solving the Dirac Equation with the Unified Transform Method
}

\author{
Casey Garner* \\ Department of Mathematics \\ Rose-Hulman Institute of Technology \\ Terre Haute, IN 47803 \\ Faculty advisor: William Green ${ }^{\dagger}$ \\ Department of Mathematics \\ Rose-Hulman Institute of Technology \\ Terre Haute, IN 47803
}

\section{Abstract}

In this article we use the Unified Transform Method to study boundary value problems for a hyperbolic system of partial differential equations from relativistic quantum mechanics. Specifically, we derive solutions to the Dirac equation in both the massive and massless cases on the half-line and the finite interval using this method.

\section{Introduction}

In this paper we study a family of boundary value problems for the Dirac equation in one spatial dimension,

$$
\left\{\begin{array}{l}
i \partial_{t} \Psi_{1}(x, t)=-i \partial_{x} \Psi_{1}(x, t)+m \Psi_{2}(x, t) \\
i \partial_{t} \Psi_{2}(x, t)=i \partial_{x} \Psi_{2}(x, t)+m \Psi_{1}(x, t)
\end{array} \quad x \in \mathbb{R}, t \geq 0\right.
$$

In particular we study the boundary value problems for this system on the half-line $x \in[0, \infty)$ and the finite interval $[0, L]$. We show the Unified Transform Method may be applied to arrive at explicit solutions determined by initial and boundary conditions.

The Dirac equation is a hyperbolic system of partial differential equations developed by Dirac to model quantum mechanical particles moving at relativistic speeds. In (1), $\Psi_{1}$ and $\Psi_{2}$ are wave functions and $m \geq 0$ is the mass of the quantum mechanical particle.

*garnercd@rose-hulman.edu

†'green@rose-hulman.edu 
We distinguish the massive $m>0$ and massless $m=0$ cases, as the underlying mathematical structure is more complicated for the massive case.

Paul Dirac derived his equation in the 1920's and it has been instrumental in various advances in particle physics in addition to having great predictive power in this area. For example, the Dirac equation implied the existence of antimatter and helped lead to the discovery of the positron, the antiparticle of the electron $[1,22,8]$. We view the system as a relativistic modification of the Schrödinger equation [3, 18, 17]. Due to the complicated structure of the Dirac equation, (1), compared to the Schrödinger or Klein-Gordon equation, the Dirac equation is far less studied. We refer the reader to the excellent text of Thaller, [21] for a more thorough introduction.

The Dirac equation can be solved by classical methods such as Fourier series and the method of characteristics, but in this paper we employ the Unified Transform Method (UTM) which is also known as Fokas's Method in the literature. The UTM is more powerful than the aforementioned classical methods because it is more applicable. For example, one cannot solve the Dirac equation on the half-line with Fourier series or very well for the massive case. Fokas and collaborators developed the method beginning in the 1990's while studying the linear version of the Korteweg-de Vries equation through an appropriate transform method $[11,15]$. Many of the key ideas of the UTM were presented in 1997 in [10]. The method has been shown to be adaptable to linear and certain non-linear problems, see for example $[10,12]$. We note that boundary value problems for the Schrödinger equation have been well studied with the UTM, see $[2,13]$ among others. Just recently, the method was applied to a general class of systems of PDEs [4].

The UTM has many properties of interest. One, the UTM can handle complicated domains more readily than classical methods where different implementations are necessary for different domains. Domains with interfaces and various other examples can be found in $[5,6]$. More applications of the UTM are available at the website [20] which contains a large compilation of published articles on the method. The Unified Transform Method also has the ability to provide information about the number and type of boundary conditions necessary for well-posed problems. Additionally, the UTM has better computational properties that reduce the Gibbs phenomenon at the boundaries. For more on the numerical properties of the UTM see $[9,16]$.

The UTM follows a general procedure when solving scalar PDEs and systems of linear PDEs. A list of the steps for the linear and system cases are provided in [7] and [4]. The main steps of the process include: computing the dispersion relations, determining the local relations, utilizing Green's Theorem to obtain global relations, applying the Fourier transform to compute an expression for the unknown variables, and lastly utilizing deformations in the complex plane and discrete symmetries to remove unknown constraints to arrive at a solution. 
The paper is organized as follows. We first consider the Dirac equation on the half-line in Section 2. The massless and massive systems are considered in Subsections 2.1 and 2.2 respectively. We then consider the problem on the finite interval in Section 3. Again considering the massless and massive cases in Subsections 3.1 and 3.2.

\section{Solving on the Half-Line}

Consider the Dirac equation on the half-line with Dirichlet boundary conditions,

$$
\left\{\begin{aligned}
i & \partial_{t} \Psi_{1}(x, t)=-i \partial_{x} \Psi_{1}(x, t)+m \Psi_{2}(x, t), \\
& i \partial_{t} \Psi_{2}(x, t)=i \partial_{x} \Psi_{2}(x, t)+m \Psi_{1}(x, t), \quad x \in[0, \infty), t \in(0, T], \\
& \Psi_{1}(x, 0)=\Psi_{1,0}(x), \quad \Psi_{1}(0, t)=b_{1}(t), \\
& \Psi_{2}(x, 0)=\Psi_{2,0}(x), \quad \Psi_{2}(0, t)=b_{2}(t),
\end{aligned}\right.
$$

where we assume $\Psi_{1}(x, t), \Psi_{2}(x, t) \rightarrow 0$ sufficiently fast as $x \rightarrow \infty$ for all times $t, T$ is a positive, finite time, and $m \geq 0$ is the mass of the system. To apply the Unified Transform Method to linear systems of partial differential equations, we follow the approach outlined in [4] and rewrite (2) in the form,

$$
\partial_{t} Q+\Lambda\left(-i \partial_{x}\right) Q=0 .
$$

Here $Q$ is an $N$-dimensional vector and $\Lambda$ is an $N \times N$ matrix-valued polynomial; $N=2$ for the one-dimensional Dirac system. We now re-write (2) in matrix form as,

$$
\partial_{t}\left(\begin{array}{l}
\Psi_{1} \\
\Psi_{2}
\end{array}\right)+\left(\begin{array}{cc}
\partial_{x} & m i \\
m i & -\partial_{x}
\end{array}\right)\left(\begin{array}{l}
\Psi_{1} \\
\Psi_{2}
\end{array}\right)=0
$$

Thus, from (3) we see

$$
Q=\left(\begin{array}{l}
\Psi_{1}(x, t) \\
\Psi_{2}(x, t)
\end{array}\right), \quad \Lambda(k)=\left(\begin{array}{cc}
i k & m i \\
m i & -i k
\end{array}\right) .
$$

The main distinction between the massive $(m>0)$ and massless $(m=0)$ systems is the level of complexity of the system of partial differential equations. When $m=0$, $\Lambda$ becomes a diagonal matrix and simplifies the solution process because the system of equations becomes decoupled. In the massless case, the problem reduces to two scalar partial differential equations; however, $m>0$ ensures the system is coupled and $\Lambda$ is not a diagonal matrix. For these reasons the massive system is more complicated than the massless; therefore, we solve the simpler massless case to introduce the technique before solving the massive case.

\subsection{The Massless System on the Half-line}

To solve the massless system, we set $m=0$ in (4) giving,

$$
\partial_{t}\left(\begin{array}{l}
\Psi_{1} \\
\Psi_{2}
\end{array}\right)+\left(\begin{array}{cc}
\partial_{x} & 0 \\
0 & -\partial_{x}
\end{array}\right)\left(\begin{array}{l}
\Psi_{1} \\
\Psi_{2}
\end{array}\right)=0
$$


and

$$
\Lambda(k)=\left(\begin{array}{cc}
i k & 0 \\
0 & -i k
\end{array}\right) .
$$

Before we begin, we inform the reader the massless case is overdetermined because the Dirichlet data for $\Psi_{2}$ cannot be specified. One of the key aspects of the UTM is it details which boundary conditions are necessary for a well-posed problem. By applying the UTM, we show the initial conditions of $\Psi_{2}$ dictate the Dirichlet data for $\Psi_{2}$ thus making it impossible to independently prescribe these boundary conditions.

Now, the first step to compute the solution to the massless system is calculating the dispersion relations. Computing the dispersion relations in the systems case is analogous to applying the Unified Transform Method on scalar PDEs, see [7, 4]. However, unlike the scalar case where the dispersion relation is computed by solving the adjoint problem or by substituting $e^{i k x-\omega(k) t}$ into the PDE following the convention given in [7], we let

$$
Q=\left(\begin{array}{l}
Q_{1} \\
Q_{2}
\end{array}\right) e^{i k x-\omega(k) t}
$$

Then, in the system equivalent to solving for $w(k)$ after the substitution in the scalar case, we find $\omega(k)$ such that,

$$
\operatorname{det}(\Lambda(k)-\omega(k) I)=0 .
$$

Plugging in the substitution for $Q$ after defining $\Lambda(k)$ as in (6), computing the determinant, and solving for $\omega(t)$, we determine the branches for the massless system are,

$$
\Omega_{1,2}= \pm i k .
$$

The next step in the scalar case is to determine the local relation. This remains the same for systems, but the computation is altered. In this situation, we must rewrite (3) in divergence form (see the appendix of [14]),

$$
\left(e^{-i k x I+\Omega(k) t} A(k) Q\right)_{t}-\left(e^{-i k x I+\Omega(k) t} A(k) X(x, t, k) Q\right)_{x}=0,
$$

where $\Omega(k)=\operatorname{diag}\left(\Omega_{1}, \ldots, \Omega_{N}\right)$ is a diagonal matrix of the different branches of the system and $A(k)$ diagonalizes $\Lambda(k)$, that is,

$$
\Lambda(k)=A^{-1}(k) \Omega(k) A(k) .
$$

Following [4], matrix $X(x, t, k)$ is defined by,

$$
X(x, t, k)=\frac{i}{k+i \partial_{x}}\left(\Lambda(k)-\Lambda\left(-i \partial_{x}\right)\right) .
$$

For the massless Dirac equation, since $\Psi(k)=\Omega(k)$ and $A(k)=I$, we have,

$$
X(x, t, k)=\frac{i}{k+i \partial_{x}}\left(\begin{array}{cc}
i k-\partial_{x} & 0 \\
0 & -i k+\partial_{x}
\end{array}\right)=\left(\begin{array}{cc}
-1 & 0 \\
0 & 1
\end{array}\right) .
$$


It should be noted the simple form of $X$ comes from the fact when $m=0$ the system is decoupled. This is not the case for the massive system. From (8), we now compute the local relations for the massless Dirac equation,

$$
\begin{aligned}
& \left(e^{-i k x+\Omega_{1} t} \Psi_{1}\right)_{t}-\left(e^{-i k x+\Omega_{1} t}\left(-\Psi_{1}\right)\right)_{x}=0, \\
& \left(e^{-i k x+\Omega_{2} t} \Psi_{2}\right)_{t}-\left(e^{-i k x+\Omega_{2} t} \Psi_{2}\right)_{x}=0 .
\end{aligned}
$$

The next step is computing the global relations from each of the local relations. In [4] a generalized expression to compute the global relations was given; however, the unknown variables in this problem are sufficiently decoupled so the scalar process can be employed to determine the global relations for (10). Computing the global relations is done by integrating each local relation over the domain, $R=\{(x, t): x \in[0, \infty), t \in(0, T]\}$, with Green's theorem.

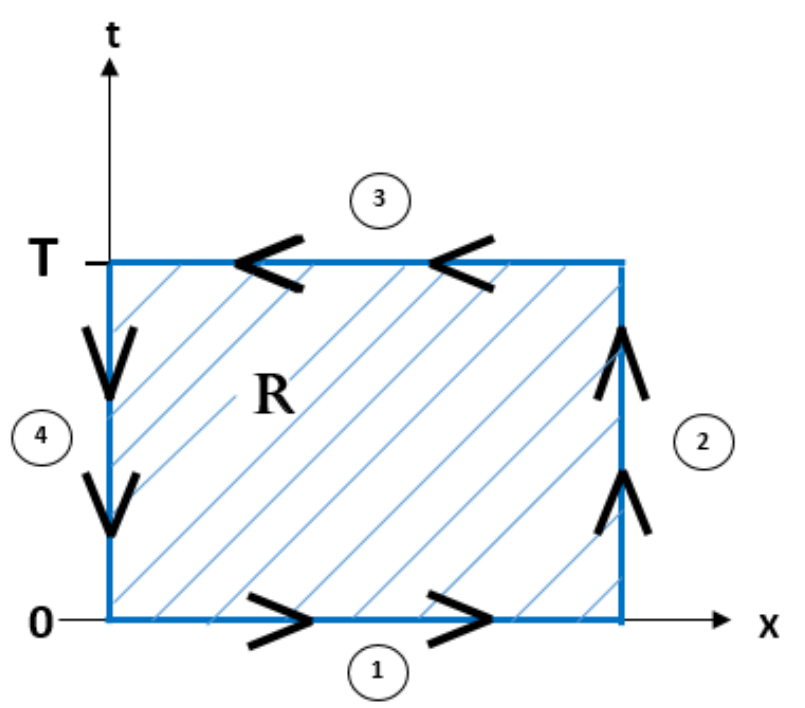

Figure 1: Domain of integration for computing the global relation with the labeled parameterizations for the necessary line integrals after application of Green's Theorem.

We first compute the global relation for $\Psi_{1}$. Integrate the first equation in (10) over $R$,

$$
\iint_{R}\left[e^{-i k x+\Omega_{1} t} \Psi_{1}\right]_{t}-\left[e^{-i k x+\Omega_{1} t}\left(-\Psi_{1}\right)\right]_{x} d t d x=0 .
$$

By invoking Green's Theorem we move the integration to the boundary of $R$,

$$
\int_{\partial R}\left[e^{-i k x+\Omega_{1} t} \Psi_{1}\right] d x+\left[e^{-i k x+\Omega_{1} t}\left(-\Psi_{1}\right)\right] d t=0 .
$$


Parameterizing the borders of the domain and integrating the respective line integrals leads to the global relation. The form of the global relation is given in terms of the Fourier transform of the unknown variable and its initial conditions. In this paper we use the following definitions of the Fourier transform and inverse Fourier transform,

$$
\begin{gathered}
\mathcal{F}\{f(x)\}=\hat{f}(k)=\int_{-\infty}^{\infty} e^{-i k x} f(x) d x, \\
\mathcal{F}^{-1}\{\hat{f}(k)\}=f(x)=\frac{1}{2 \pi} \int_{-\infty}^{\infty} e^{i k x} \hat{f}(k) d k .
\end{gathered}
$$

The contribution of the second line integral is zero because $\Psi_{1}(x, t) \rightarrow 0$ sufficiently fast as $x \rightarrow \infty$ for all $t$ and the line integral is over a finite interval since $T$ is finite. Thus, the first, second, and third terms in the global relation below come from the first, third, and fourth line integrals respectively.

$$
\hat{\Psi}_{1}(k, 0)-e^{\Omega_{1} T} \hat{\Psi}_{1}(k, T)+g_{0}\left(\Omega_{1}, b_{1}, T\right)=0,
$$

where $\hat{\Psi}_{1}(k, t)$ is the Fourier transform of $\Psi_{1}(x, t)$. We also define the variables $g_{0}\left(\Omega_{j}, b_{j}, t\right)$, $j \in\{1,2\}$, which depend on the boundary conditions as,

$$
g_{0}\left(\Omega_{j}, b_{j}, t\right)=\int_{0}^{t} e^{\Omega_{j} s} \Psi_{j}(0, s) d s=\int_{0}^{t} e^{\Omega_{j} s} b_{j}(s) d s .
$$

We also note the half-line definition of the Fourier transform is utilized in computation because the values of $\Psi_{1}(x, t)$ and $\Psi_{2}(x, t)$ are assumed zero for all $(x, t)$ outside the domain $R$. Following the same procedure for $\Psi_{2}$ leads to the second global relation,

$$
\hat{\Psi}_{2}(k, 0)-e^{\Omega_{2} T} \hat{\Psi}_{2}(k, T)-g_{0}\left(\Omega_{2}, b_{2}, T\right)=0 .
$$

We can replace $T$ in the global relations with any $t \in[0, T]$ because both equations are valid for all feasible values of $t$. Thus, the global relations for the massless case are,

$$
\begin{aligned}
& \hat{\Psi}_{1}(k, 0)-e^{\Omega_{1} t} \hat{\Psi}_{1}(k, t)+g_{0}\left(\Omega_{1}, b_{1}, t\right)=0, \\
& \hat{\Psi}_{2}(k, 0)-e^{\Omega_{2} t} \hat{\Psi}_{2}(k, t)-g_{0}\left(\Omega_{2}, b_{2}, t\right)=0 .
\end{aligned}
$$

The next step is to utilize the inverse Fourier transform to obtain expressions for $\Psi_{1}(x, t)$ and $\Psi_{2}(x, t)$ from (12). Multiplying the first equation in (12) by $e^{-\Omega_{1} t}$ and the second by $e^{-\Omega_{2} t}$, then algebraically solving for the Fourier transforms yields

$$
\begin{aligned}
& \hat{\Psi}_{1}(k, t)=e^{-\Omega_{1} t}\left(\hat{\Psi}_{1,0}(k)+g_{0}\left(\Omega_{1}, b_{1}, t\right)\right), \\
& \hat{\Psi}_{2}(k, t)=e^{-\Omega_{2} t}\left(\hat{\Psi}_{2,0}(k)-g_{0}\left(\Omega_{2}, b_{2}, t\right)\right) .
\end{aligned}
$$


Taking the inverse Fourier transforms of the above expressions and substituting in the dispersion relations $(7)$,

$$
\begin{aligned}
& \Psi_{1}(x, t)=\frac{1}{2 \pi} \int_{-\infty}^{\infty} e^{i k(x-t)}\left(\hat{\Psi}_{1,0}(k)+g_{0}\left(\Omega_{1}, b_{1}, t\right)\right) d k \\
& \Psi_{2}(x, t)=\frac{1}{2 \pi} \int_{-\infty}^{\infty} e^{i k(x+t)}\left(\hat{\Psi}_{2,0}(k)-g_{0}\left(\Omega_{2}, b_{2}, t\right)\right) d k .
\end{aligned}
$$

Now, (13) is not a solution because the expressions are overdetermined. So, our next step is to remove the unnecessary Dirichlet boundary condition of $\Psi_{2}$ and simplify (13) to arrive at the solution. Normally when applying Fokas's Method one accomplishes this through contours. In this case, the dispersion relations have zero non-trivial discrete symmetries, so we use analytic properties to simplify (13). Expanding (13) with the definitions for $g_{0}\left(\Omega_{j}, b_{j}, t\right),(11)$,

$$
\begin{aligned}
& \Psi_{1}(x, t)=\frac{1}{2 \pi} \int_{-\infty}^{\infty} e^{i k(x-t)} \hat{\Psi}_{1,0}(k) d k+\frac{1}{2 \pi} \int_{-\infty}^{\infty} \int_{0}^{t} e^{i k(x-t+s)} b_{1}(s) d s d k, \\
& \Psi_{2}(x, t)=\frac{1}{2 \pi} \int_{-\infty}^{\infty} e^{i k(x+t)} \hat{\Psi}_{2,0}(k) d k-\frac{1}{2 \pi} \int_{-\infty}^{\infty} \int_{0}^{t} e^{i k(x+t-s)} b_{2}(s) d s d k .
\end{aligned}
$$

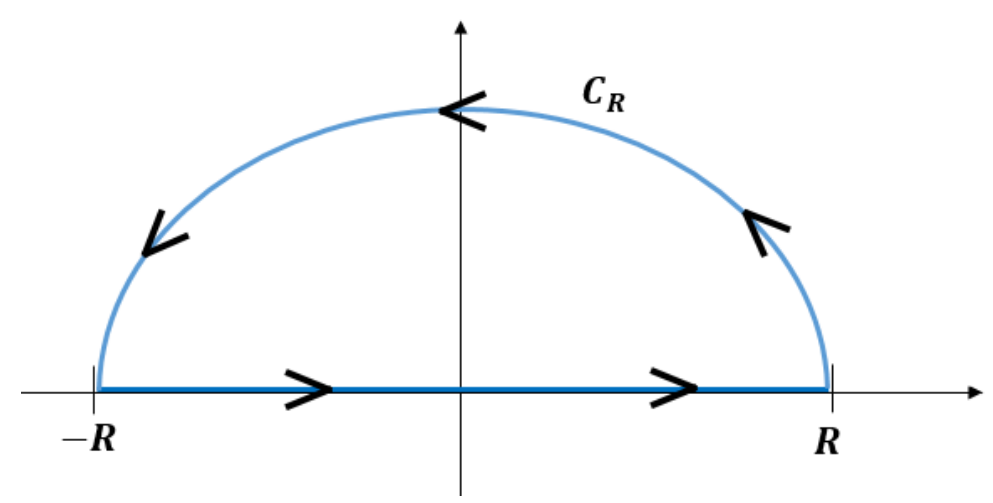

Figure 2: Contour used to remove the boundary conditions for $\Psi_{2}(x, t)$ in (14)

In the second equation of (14), we see the integrand for the second integral over the real line is analytic. Since $x+t-s>0$, if we integrate

$$
\int_{0}^{t} e^{i k(x+t-s)} b_{2}(s) d s
$$

along the contour $C=[-R, R] \cup C_{R}$ where $C_{R}$ is the circular arc of radius $R$ centered at the origin in $\mathbb{C}^{+}$, by Cauchy's Theorem,

$$
\left[\int_{-R}^{R}+\int_{C_{R}}\right]\left(\int_{0}^{t} e^{i k(x+t-s)} b_{2}(s) d s\right) d k=0 .
$$


Taking the limit as $R \rightarrow \infty$ and applying Jordan's Lemma, due to the exponential decay, the integral along $C_{R}$ goes to 0 ; therefore,

$$
\int_{-\infty}^{\infty} \int_{0}^{t} e^{i k(x+t-s)} b_{2}(s) d s d k=0
$$

and using Fourier inversion,

$$
\Psi_{2}(x, t)=\frac{1}{2 \pi} \int_{-\infty}^{\infty} e^{i k(x+t)} \hat{\Psi}_{2,0}(k) d k=\Psi_{2,0}(x+t) .
$$

To simplify $\Psi_{1}(x, t)$ in (14) we work in cases.

Case 1: If $x>t$, then $x-t+s>0$ and using the previous arguments,

$$
\Psi_{1}(x, t)=\frac{1}{2 \pi} \int_{-\infty}^{\infty} e^{i k(x-t)} \hat{\Psi}_{1,0}(k) d k=\Psi_{1,0}(x-t)
$$

Case 2: If $x<t$, then $x-t+s<0$ and we cannot use contour $C$ to eliminate the second integral defining $\Psi_{1}(x, t)$; however, using the distributional definition of the delta distribution we see,

$$
\frac{1}{2 \pi} \int_{-\infty}^{\infty} \int_{0}^{t} e^{i k(x-t+s)} b_{1}(s) d s d k=b_{1}(t-x) .
$$

So, for $x<t$,

$$
\Psi_{1}(x, t)=b_{1}(t-x)
$$

by the definition of the inverse Fourier transform and $\Psi_{1,0}(x-t)=0$ since $x-t<0$.

In this paper, we assume all boundary and initial conditions are compatible whenever they interface; therefore, in this problem when $x=t, \Psi_{1,0}(x-t)=b_{1}(t-x)$.

Remark: It should be noted the delta distribution argument in Case 2 could have been utilized for Case 1 instead of using contour $C$; however, this is only due to the simple dispersion relation (7) for the massless Dirac equation. Since invoking the delta distribution does not generalize to determining the massive solution we use the contour argument.

These simplifications give the solution to the massless Dirac equation on the half-line,

$$
\begin{aligned}
& \Psi_{1}(x, t)=\left\{\begin{array}{cc}
\Psi_{1,0}(x-t), & x \geq t, \\
b_{1}(t-x), & x<t
\end{array}\right. \\
& \Psi_{2}(x, t)=\Psi_{2,0}(x+t)
\end{aligned}
$$


Notice, since $\Psi_{1,0}(x-t)=b_{1}(t-x)$ when $x=t$ the equality in (15) could have went on either case. Now, the form of these solutions were expected because the massless case on the half-line for (2) reduces to the transport equation for $\Psi_{1}(x, t)$ and $\Psi_{2}(x, t)$. The method of characteristics gives the solution to the transport equation as left and right moving waves agreeing with (15). The two different cases correspond with how the boundary conditions and initial conditions propagate. The boundary and initial conditions affect disjoint sections of the domain because information travels along the characteristics. In contrast to these simple solutions, the massive case is more challenging and requires additional work to remove unnecessary terms containing the Dirichlet boundary conditions.

\subsection{Massive System $(m>0)$}

Now we solve the massive problem on the half-line. The mass term will now be some positive real number, so we are solving,

$$
\partial_{t}\left(\begin{array}{l}
\Psi_{1} \\
\Psi_{2}
\end{array}\right)+\left(\begin{array}{cc}
\partial_{x} & i m \\
i m & -\partial_{x}
\end{array}\right)\left(\begin{array}{l}
\Psi_{1} \\
\Psi_{2}
\end{array}\right)=0
$$

and

$$
\Lambda(k)=\left(\begin{array}{cc}
i k & i m \\
i m & -i k
\end{array}\right) .
$$

With non-zero mass, the system of PDEs is now coupled. Thus, in this case (2) is not overdetermined, but the UTM will show in the coming steps that the solutions to $\Psi_{1}$ and $\Psi_{2}$ do not depend on each other's boundary conditions. In other words, the solution for $\Psi_{1}$ depends on both initial conditions and its Dirichlet condition but not the boundary condition of $\Psi_{2}$ and vice versa for $\Psi_{2}$.

Now, we begin again by computing the dispersion relation. The computation follows as before in the massless case. We find $\omega(k)$ such that,

$$
\operatorname{det}(\Lambda(k)-\omega(k) I)=0 .
$$

$\Lambda(k)$ is no longer a diagonal matrix which adds complexity to the dispersion relation. Performing the calculations, the branches for the massive system are,

$$
\Omega_{1,2}= \pm i \sqrt{k^{2}+m^{2}} .
$$

Obtaining the local relation for the massive system still requires the system to be in divergence form given by,

$$
\left(e^{-i k x I+\Omega(k) t} A(k) Q\right)_{t}-\left(e^{-i k x I+\Omega(k) t} A(k) X(x, t, k) Q\right)_{x}=0 .
$$

The value of $X(x, t, k)$ does not change from the massless case (9), but the diagonalization of $\Lambda(k)$ is different due to the different branches. For the massive case,

$$
A=\left(\begin{array}{cc}
i m & \Omega_{1}-i k \\
i m & \Omega_{2}-i k
\end{array}\right), \quad X=\left(\begin{array}{cc}
-1 & 0 \\
0 & 1
\end{array}\right) \text {. }
$$


With these definitions, after simplifying we obtain the local relations for $j \in\{1,2\}$,

$$
\left(e^{-i k x+\Omega_{j} t}\left[(i m) \Psi_{1}+\left(\Omega_{j}-i k\right) \Psi_{2}\right]\right)_{t}-\left(e^{-i k x+\Omega_{j} t}\left[(-i m) \Psi_{1}+\left(\Omega_{j}-i k\right) \Psi_{2}\right]\right)_{x}=0 .
$$

The global relations for the massive case are determined in the same manner as before in the massless case. We first integrate the local relations over the entire domain, $R$,

$$
\begin{aligned}
\iint_{R}\left(e^{-i k x+\Omega_{j} t}\left[(i m) \Psi_{1}+\left(\Omega_{j}-i k\right) \Psi_{2}\right]\right)_{t} \\
-\left(e^{-i k x+\Omega_{j} t}\left[(-i m) \Psi_{1}+\left(\Omega_{j}-i k\right) \Psi_{2}\right]\right)_{x} d x d t=0
\end{aligned}
$$

apply Green's Theorem to move the integration to the boundary,

$$
\begin{aligned}
\int_{\partial R}\left(e^{-i k x+\Omega_{j} t}\left[(i m) \Psi_{1}+\left(\Omega_{j}-i k\right) \Psi_{2}\right]\right) d x & \\
& +\left(e^{-i k x+\Omega_{j} t}\left[(-i m) \Psi_{1}+\left(\Omega_{j}-i k\right) \Psi_{2}\right]\right) d t=0
\end{aligned}
$$

and lastly parameterize the $\partial R$ and use the properties of $\Psi_{1}$ and $\Psi_{2}$ to compute the various line integrals. Performing the integration allows us to the write the two global relations. The global relations for the massive case are,

$$
\begin{aligned}
(i m) \hat{\Psi}_{1}(k, 0) & +\left(\Omega_{j}-i k\right) \hat{\Psi}_{2}(k, 0)+(-i m) e^{\Omega_{j} t} \hat{\Psi}_{1}(k, t) \\
& +\left(i k-\Omega_{j}\right) e^{\Omega_{j} t} \hat{\Psi}_{2}(k, t)+(i m) h_{0,1}\left(\Omega_{j}, t\right)+\left(i k-\Omega_{j}\right) h_{0,2}\left(\Omega_{j}, t\right)=0,
\end{aligned}
$$

for $j \in\{1,2\}$ where $T$ has been replaced with $t$ and

$$
h_{0, l}\left(\Omega_{j}, t\right)=\int_{0}^{t} e^{\Omega_{j} s} \Psi_{l}(0, s) d s=\int_{0}^{t} e^{\Omega_{j} s} b_{l}(s) d s .
$$

In order to apply the inverse Fourier transform, we take the two global relations and solve for $\hat{\Psi}_{1}(k, t)$ and $\hat{\Psi}_{2}(k, t)$. We multiply equation (17), with $j=1$ and $j=2$, by $e^{-\Omega_{1} t}$ and $e^{-\Omega_{2} t}$ respectively to obtain,

$$
\begin{aligned}
& (-i m) \hat{\Psi}_{1}(k, t)+\left(i k-\Omega_{1}\right) \hat{\Psi}_{2}(k, t)+C_{1}(k, t) e^{-\Omega_{1} t}=0, \\
& (-i m) \hat{\Psi}_{1}(k, t)+\left(i k-\Omega_{2}\right) \hat{\Psi}_{2}(k, t)+C_{2}(k, t) e^{-\Omega_{2} t}=0,
\end{aligned}
$$

where

$C_{j}(k, t)=(i m) \hat{\Psi}_{1,0}(k)+\left(\Omega_{j}-i k\right) \hat{\Psi}_{2,0}(k)+(i m) h_{0,1}\left(\Omega_{j}, t\right)+\left(i k-\Omega_{j}\right) h_{0,2}\left(\Omega_{j}, t\right), \quad j \in\{1,2\}$.

Subtracting the second equation in (19) from the first, and solving for $\hat{\Psi}_{2}(k, t)$, using the fact $\Omega_{2}-\Omega_{1}=2 \Omega_{2}$, yields,

$$
\hat{\Psi}_{2}(k, t)=\frac{1}{2 \Omega_{2}}\left(C_{2}(k, t) e^{-\Omega_{2} t}-C_{1}(k, t) e^{-\Omega_{1} t}\right) .
$$


Adding both equations in (19), substituting (20), and solving for $\hat{\Psi}_{1}(k, t)$,

$$
\hat{\Psi}_{1}(k, t)=\left(\frac{\Omega_{2} i+k}{2 m \Omega_{1}}\right) C_{1}(k, t) e^{-\Omega_{1} t}+\left(\frac{\Omega_{2} i-k}{2 m \Omega_{1}}\right) C_{2}(k, t) e^{-\Omega_{2} t} .
$$

Taking the inverse Fourier transforms of (20) and (21) we obtain:

$$
\begin{gathered}
\Psi_{1}(x, t)=\frac{1}{4 m \pi} \int_{-\infty}^{\infty}\left(\frac{\Omega_{2} i+k}{\Omega_{1}}\right) C_{1}(k, t) e^{i k x-\Omega_{1} t} d k \\
+\frac{1}{4 m \pi} \int_{-\infty}^{\infty}\left(\frac{\Omega_{2} i-k}{\Omega_{1}}\right) C_{2}(k, t) e^{i k x-\Omega_{2} t} d k, \\
\Psi_{2}(x, t)=\frac{1}{4 \pi} \int_{-\infty}^{\infty} \frac{e^{i k x-\Omega_{2} t}}{\Omega_{2}} C_{2}(k, t) d k-\frac{1}{4 \pi} \int_{-\infty}^{\infty} \frac{e^{i k x-\Omega_{1} t}}{\Omega_{2}} C_{1}(k, t) d k
\end{gathered}
$$

Both equations are overdetermined because each contains Dirichlet boundary conditions which are unnecessary. The first expression contains the boundary condition for $\Psi_{2}$, and the second expression contains the boundary condition for $\Psi_{1}$. Both are superfluous to their respective expressions, and to remedy this we utilize the discrete symmetry of the dispersion relations, $k \rightarrow-k$. Note, $\Omega_{1,2}(k)$ are invariant under $\nu(k)=-k$ since $\Omega_{1,2}(k)=\Omega_{1,2}(\nu(k))$. Applying this transformation to (21) and (20) we have,

$$
\begin{gathered}
\hat{\Psi}_{1}(-k, t)=\left(\frac{\Omega_{2} i-k}{2 m \Omega_{1}}\right) C_{1}(-k, t) e^{-\Omega_{1} t}+\left(\frac{\Omega_{2} i+k}{2 m \Omega_{1}}\right) C_{2}(-k, t) e^{-\Omega_{2} t}, \\
\hat{\Psi}_{2}(-k, t)=\frac{1}{2 \Omega_{2}}\left(C_{2}(-k, t) e^{-\Omega_{2} t}-C_{1}(-k, t) e^{-\Omega_{1} t}\right) .
\end{gathered}
$$

Taking the inverse Fourier transform of (24) and (25) and grouping all the terms on one-side yields expressions which sum to zero. Adding these expressions to (22) and (23) yields:

$$
\begin{aligned}
\Psi_{1}(x, t)= & \frac{1}{4 m \pi} \int_{-\infty}^{\infty} \frac{e^{i k x-\Omega_{1} t}}{\Omega_{1}}\left[m \Omega_{1}\left(\hat{\Psi}_{1,0}(k)-\hat{\Psi}_{1,0}(-k)\right)+i m k\left(\hat{\Psi}_{1,0}(k)+\hat{\Psi}_{1,0}(-k)\right)\right. \\
& \left.+\left(i k^{2}+i \Omega_{1}^{2}\right)\left(\hat{\Psi}_{2,0}(-k)-\hat{\Psi}_{2,0}(k)\right)+(2 i m k) h_{0,1}\left(\Omega_{1}, t\right)\right] d k \\
+\frac{1}{4 m \pi} \int_{-\infty}^{\infty} \frac{e^{i k x-\Omega_{2} t}}{\Omega_{1}}\left[m \Omega_{1}\left(\hat{\Psi}_{1,0}(k)-\hat{\Psi}_{1,0}(-k)\right)-i m k\left(\hat{\Psi}_{1,0}(k)+\hat{\Psi}_{1,0}(-k)\right)\right. & \\
& \left.+\left(i \Omega_{1} \Omega_{2}-i k^{2}\right)\left(\hat{\Psi}_{2,0}(-k)-\hat{\Psi}_{2,0}(k)\right)-(2 i m k) h_{0,1}\left(\Omega_{2}, t\right)\right] d k \\
+ & \frac{1}{2 \pi} \int_{-\infty}^{\infty} e^{i k x} \hat{\Psi}_{1}(-k, t) d k
\end{aligned}
$$




$$
\begin{gathered}
\Psi_{2}(x, t)=\frac{1}{4 \pi} \int_{-\infty}^{\infty} \frac{e^{i k x-\Omega_{2} t}}{\Omega_{2}}\left[i m\left(\hat{\Psi}_{1,0}(k)-\hat{\Psi}_{1,0}(-k)\right)+\Omega_{2}\left(\hat{\Psi}_{2,0}(k)-\hat{\Psi}_{2,0}(-k)\right)\right. \\
\left.-i k\left(\hat{\Psi}_{2,0}(k)-\hat{\Psi}_{2,0}(-k)\right)+(2 i k) h_{0,2}\left(\Omega_{2}, t\right)\right] d k \\
-\frac{1}{4 \pi} \int_{-\infty}^{\infty} \frac{e^{i k x-\Omega_{1} t}}{\Omega_{2}}\left[i m\left(\hat{\Psi}_{1,0}(k)-\hat{\Psi}_{1,0}(-k)\right)+\Omega_{1}\left(\hat{\Psi}_{2,0}(k)-\hat{\Psi}_{2,0}(-k)\right)\right. \\
\left.\quad-i k\left(\hat{\Psi}_{2,0}(k)-\hat{\Psi}_{2,0}(-k)\right)+(2 i k) h_{0,2}\left(\Omega_{1}, t\right)\right] d k \\
+\frac{1}{2 \pi} \int_{-\infty}^{\infty} e^{i k x} \hat{\Psi}_{2}(-k, t) d k,
\end{gathered}
$$

where $h_{0,1}$ and $h_{0,2}$, defined in (18), contain the Dirichlet boundary conditions, and $\hat{\Psi}_{1,0}$ and $\hat{\Psi}_{2,0}$ are the Fourier transforms of the initial conditions for $\Psi_{1}$ and $\Psi_{2}$. Notice, in (26) and (27) respectively, the boundary conditions for $\Psi_{2}$ and $\Psi_{1}$ were eliminated, removing the overdetermining boundary conditions. (26) and (27) are nearly solution formulas except for the last terms in each expression. Both terms contain the Fourier transform of an unknown variable and hence cannot be a solution; however, we can show both terms are zero by using an argument employed by Deconinck et al. in Section 8 of [4]. Note, following Section 4 in [4], we interpret the half-line transforms as whole line transforms of functions that are zero for $x<0$. For the last term in (26), by substitution we note,

$$
\frac{1}{2 \pi} \int_{-\infty}^{\infty} e^{i k x} \hat{\Psi}_{1}(-k, t) d k=\frac{1}{2 \pi} \int_{-\infty}^{\infty} e^{i k(-x)} \hat{\Psi}_{1}(k, t) d k=\Psi_{1}(-x, t)
$$

because we are taking the inverse Fourier transform of a Fourier transform. Thus, by our interpretation of the half-line transforms and since $\Psi_{1}$ and $\Psi_{2}$ are defined as zero for negative $x, \Psi_{1}(-x, t)=0$ for all $x$. It is easy to see this same argument applies to the last term in (27); therefore, the solutions for $\Psi_{1}$ and $\Psi_{2}$ are (26) and (27) with their final terms removed. It is worth noting (26) and (27) are similar in form to the solutions of the Klein-Gordon equation in Section 7 of [4]. This meshes well with the interpretation of the Dirac equation as a square root of a diagonal system of Klein-Gordon equations.

\section{Solving on the Finite Interval}

We continue to generalize the solution to the Dirac equation by solving the system on the finite interval, $[0, L]$, for $L \in \mathbb{R}^{+}$finite. We first solve the massless and then the massive systems. As before, the massive system has a more complex solution, but clear and expected consistency exists between the finite interval and half-line solutions. 
The only change in the Dirac equation set-up from the previous section is the addition of another set of Dirichlet boundary conditions at $x=L$ and $x \in[0, L]$. The finite Dirac equation is,

$$
\begin{gathered}
i \partial_{t} \Psi_{1}(x, t)=-i \partial_{x} \Psi_{1}(x, t)+m \Psi_{2}(x, t), \quad x \in[0, L], t \in(0, T], \\
i \partial_{t} \Psi_{2}(x, t)=i \partial_{x} \Psi_{2}(x, t)+m \Psi_{1}(x, t), \quad x \in[0, L], t \in(0, T], \\
\Psi_{1}(x, 0)=\Psi_{1,0}(x), \Psi_{2}(x, 0)=\Psi_{2,0}(x), \quad x \in[0, L], \\
\Psi_{1}(0, t)=\alpha_{1}(t), \Psi_{2}(0, t)=\alpha_{2}(t), \quad t \in[0, T] \\
\Psi_{1}(L, t)=\beta_{1}(t), \Psi_{2}(L, t)=\beta_{2}(t), \quad t \in[0, T]
\end{gathered}
$$

where $T$ is a positive, finite, time value, and $\alpha_{i}(t)$ and $\beta_{i}(t), i \in\{1,2\}$, are the Dirichlet boundary conditions for $\Psi_{1}(x, t)$ and $\Psi_{2}(x, t)$ at $x=0$ and $x=L$ respectively.

\subsection{Massless System $(m=0)$}

All of the previous work in the last section for the massless and massive systems on the half-line remain the same until the global relations are computed. The dispersion and local relations are not altered by the domain of the problem or the known boundary conditions; therefore, we begin the solution process for the massless Dirac equation on the finite interval at the computation of the global relations.

Analogous to Section 2.1, we inform the reader the system as given in (28) for the massless system is overdetermined. The UTM in the coming steps illuminates neither the right boundary condition for $\Psi_{1}$ nor the left boundary condition for $\Psi_{2}$ can be independently prescribed. Both of these conditions are specified by the initial conditions and the other Dirichlet conditions.

The same process is undertaken to compute the global relations. We integrate the local relation for $\Psi_{1}(x, t)$ over the domain, $R=\{(x, t): x \in[0, L], t \in[0, T]\}$,

$$
\iint_{R}\left[e^{-i k x+\Omega_{1} t} \Psi_{1}\right]_{t}-\left[e^{-i k x+\Omega_{1} t}\left(-\Psi_{1}\right)\right]_{x} d t d x=0,
$$

and apply Green's Theorem to move the integration to the boundary,

$$
\int_{\partial R}\left[e^{-i k x+\Omega_{1} t} \Psi_{1}\right] d x+\left[e^{-i k x+\Omega_{1} t}\left(-\Psi_{1}\right)\right] d t=0 .
$$

Performing the integration leads to the global relation,

$$
\hat{\Psi}_{1}(k, 0)-e^{-i k L} B_{L}\left(\Omega_{1}, t\right)-e^{\Omega_{1} t} \hat{\Psi}_{1}(k, t)+B_{0}\left(\Omega_{1}, t\right)=0,
$$

where we define,

$$
B_{0}\left(\Omega_{j}, t\right)=\int_{0}^{t} e^{\Omega_{j} s} \Psi_{j}(0, s) d s=\int_{0}^{t} e^{\Omega_{j} s} \alpha_{j}(s) d s,
$$




$$
B_{L}\left(\Omega_{j}, t\right)=\int_{0}^{t} e^{\Omega_{j} s} \Psi_{j}(L, s) d s=\int_{0}^{t} e^{\Omega_{j} s} \beta_{j}(s) d s .
$$

Following the same procedure for the local relation of $\Psi_{2}(x, t)$ gives the second global relation,

$$
\hat{\Psi}_{2}(k, 0)+e^{-i k L} B_{L}\left(\Omega_{2}, t\right)-e^{\Omega_{2} t} \hat{\Psi}_{2}(k, t)-B_{0}\left(\Omega_{2}, t\right)=0 .
$$

We now solve for $\hat{\Psi}_{1}(k, t)$ and $\hat{\Psi}_{2}(k, t)$ in $(29)$ and (32) and apply the inverse Fourier transform. Solving for the Fourier transforms,

$$
\begin{aligned}
& \hat{\Psi}_{1}(k, t)=e^{-\Omega_{1} t}\left(\hat{\Psi}_{1}(k, 0)-e^{-i k L} B_{L}\left(\Omega_{1}, t\right)+B_{0}\left(\Omega_{1}, t\right)\right), \\
& \hat{\Psi}_{2}(k, t)=e^{-\Omega_{2} t}\left(\hat{\Psi}_{2}(k, 0)+e^{-i k L} B_{L}\left(\Omega_{2}, t\right)-B_{0}\left(\Omega_{2}, t\right)\right),
\end{aligned}
$$

and taking the inverse Fourier transform of these expressions leads to,

$$
\begin{aligned}
& \Psi_{1}(x, t)=\frac{1}{2 \pi} \int_{-\infty}^{\infty} e^{i k(x-t)}\left(\hat{\Psi}_{1,0}(k)-e^{-i k L} B_{L}\left(\Omega_{1}, t\right)+B_{0}\left(\Omega_{1}, t\right)\right) d k, \\
& \Psi_{2}(x, t)=\frac{1}{2 \pi} \int_{-\infty}^{\infty} e^{i k(x+t)}\left(\hat{\Psi}_{2,0}(k)+e^{-i k L} B_{L}\left(\Omega_{2}, t\right)-B_{0}\left(\Omega_{2}, t\right)\right) d k .
\end{aligned}
$$

As in the half-line, massless case, we can simplify (33) and (34) with regards to the boundary conditions to remove the unnecessary right boundary condition of $\Psi_{1}$ and the left boundary condition of $\Psi_{2}$. Expanding (33) and (34) with definitions (30) and (31),

$$
\begin{array}{r}
\Psi_{1}(x, t)=\frac{1}{2 \pi} \int_{-\infty}^{\infty} e^{i k(x-t)} \hat{\Psi}_{1,0}(k) d k-\frac{1}{2 \pi} \int_{-\infty}^{\infty} \int_{0}^{t} e^{i k(x-t-L+s)} \beta_{1}(s) d s d k \\
+\frac{1}{2 \pi} \int_{-\infty}^{\infty} \int_{0}^{t} e^{i k(x-t+s)} \alpha_{1}(s) d s d k \\
\Psi_{2}(x, t)=\frac{1}{2 \pi} \int_{-\infty}^{\infty} e^{i k(x+t)} \hat{\Psi}_{2,0}(k) d k+\frac{1}{2 \pi} \int_{-\infty}^{\infty} \int_{0}^{t} e^{i k(x+t-L-s)} \beta_{2}(s) d s d k \\
-\frac{1}{2 \pi} \int_{-\infty}^{\infty} \int_{0}^{t} e^{i k(x+t-s)} \alpha_{2}(s) d s d k
\end{array}
$$

Simplifying (35) first, since $x<L$ and $s<t$, this implies $x-t-L+s<0$. Using a contour similar to $C$ shown in Figure 2, except with $C_{R}$ in the lower half plane in $\mathbb{C}$, we can apply Cauchy's Theorem and Jordan's Lemma to show the integral vanishes due to the exponential decay and analytic properties of the integrand. The new contour is displayed below in Figure 3. 


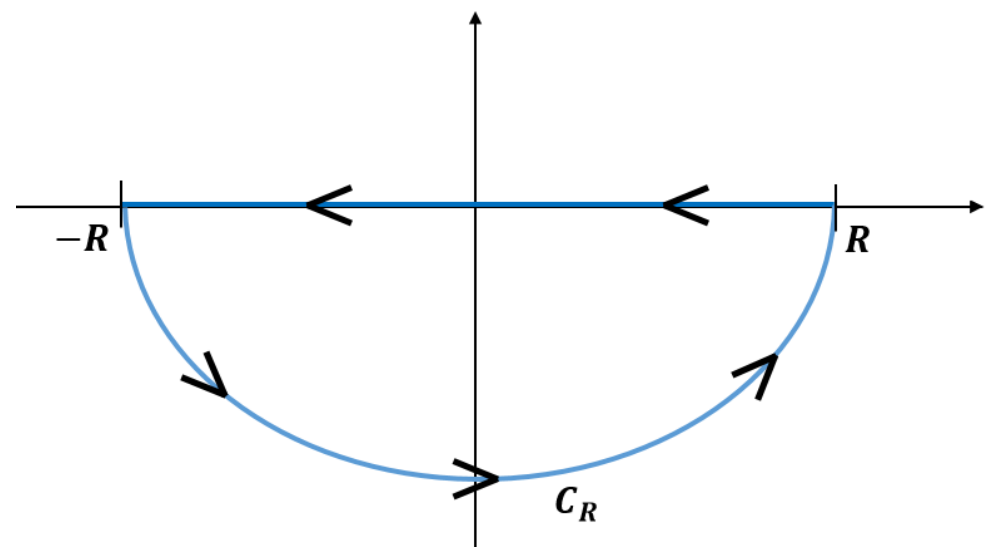

Figure 3: Contour utilized to simplify (35) which is similar to contour $C$ in previous section.

The last integral in (35) is a previous case dealt with in Subsection 2.1, so applying the inverse Fourier transform,

$$
\Psi_{1}(x, t)= \begin{cases}\Psi_{1,0}(x-t), & x \geq t, \\ \alpha_{1}(t-x), & x<t .\end{cases}
$$

In (36), the last integral was shown to vanish in Subsection 2.1 by applying contour $C$. The second integral in (36) is not signed because $t-s>0$ and $x-L<0$, so we use the definition of the delta distribution to obtain,

$$
\frac{1}{2 \pi} \int_{-\infty}^{\infty} \int_{0}^{t} e^{i k(x+t-L-s)} \beta_{2}(s) d s d k=\beta_{2}(x+t-L) .
$$

When $x+t-L<0, \beta_{2}(x+t-L)=0$ because the boundary condition is only defined for positive time values. Therefore, applying the inverse Fourier transform to these cases,

$$
\Psi_{2}(x, t)= \begin{cases}\Psi_{2,0}(x+t), & x+t \leq L, \\ \beta_{2}(x+t-L), & x+t>L\end{cases}
$$

where we have $\Psi_{2,0}(x+t)=0$ when $x+t>L$ because these arguments are outside the domain where initial conditions are specified. Therefore, the solution to the massless 
Dirac equation on the finite interval $[0, L]$ is,

$$
\begin{aligned}
& \Psi_{1}(x, t)= \begin{cases}\Psi_{1,0}(x-t), & x \geq t, \\
\alpha_{1}(t-x), & x<t\end{cases} \\
& \Psi_{2}(x, t)= \begin{cases}\Psi_{2,0}(x+t), & x+t \leq L, \\
\beta_{2}(x+t-L), & x+t>L .\end{cases}
\end{aligned}
$$

Each different case aligns with how information travels along the characteristics. $\Psi_{2}(x, t)$ does not need the left boundary specified because it is determined by the initial and right boundary conditions traveling to the left. This is also true for $\Psi_{1}(x, t)$ except the right boundary condition is determined by the initial and left boundary conditions propagating rightward.

\subsection{Massive System $(m>0)$}

For the massive system, we again start at the global relations because the dispersion and local relations are not affected by restraining the domain of the PDEs to a finite region in the plane. These properties are only dictated by local information and thus are not altered by the global situation of the partial differential equations.

Analogous to the massive case on the half-line, the system as stated for the massive case on the finite interval is not overdetermined. The final solutions computed via the UTM however will show the solution for $\Psi_{1}$ has no dependence on the left boundary condition of $\Psi_{2}$, and the solution of $\Psi_{2}$ has no dependence on the left boundary condition of $\Psi_{1}$.

Using the same process to compute the global relation as before, integrate the local relations over the domain, $R=\{(x, t): x \in[0, L], t \in[0, T]\}$,

$$
\begin{aligned}
\iint_{R}\left(e ^ { - i k x + \Omega _ { j } t } \left[(i m) \Psi_{1}+\right.\right. & \left.\left.\left(\Omega_{j}-i k\right) \Psi_{2}\right]\right)_{t} \\
& -\left(e^{-i k x+\Omega_{j} t}\left[(-i m) \Psi_{1}+\left(\Omega_{j}-i k\right) \Psi_{2}\right]\right)_{x} d x d t=0
\end{aligned}
$$

and apply Green's Theorem to move the integration to the boundary,

$$
\begin{aligned}
\int_{\partial R}\left(e^{-i k x+\Omega_{j} t}\left[(i m) \Psi_{1}+\left(\Omega_{j}-i k\right) \Psi_{2}\right]\right) d x & \\
& +\left(e^{-i k x+\Omega_{j} t}\left[(-i m) \Psi_{1}+\left(\Omega_{j}-i k\right) \Psi_{2}\right]\right) d t=0 .
\end{aligned}
$$


Performing the necessary line integrals gives the following global relations,

$$
\begin{aligned}
(i m) \hat{\Psi}_{1}(k, 0) & +\left(\Omega_{j}-i k\right) \hat{\Psi}_{2}(k, 0) \\
& +(-i m) e^{-i k L} B_{L, 1}\left(\Omega_{j}, t\right)+\left(\Omega_{j}-i k\right) e^{-i k L} B_{L, 2}\left(\Omega_{j}, t\right) \\
& +(-i m) e^{\Omega_{j} t} \hat{\Psi}_{1}(k, t)+\left(i k-\Omega_{j}\right) e^{\Omega_{j} t} \hat{\Psi}_{2}(k, t) \\
& +(i m) B_{0,1}\left(\Omega_{j}, t\right)+\left(i k-\Omega_{j}\right) B_{0,2}\left(\Omega_{j}, t\right)=0,
\end{aligned}
$$

for $j \in\{1,2\}$ where we define,

$$
\begin{aligned}
& B_{0, i}\left(\Omega_{j}, t\right)=\int_{0}^{t} e^{\Omega_{j} s} \Psi_{i}(0, s) d s=\int_{0}^{t} e^{\Omega_{j} s} \alpha_{i}(s) d s \\
& B_{L, i}\left(\Omega_{j}, t\right)=\int_{0}^{t} e^{\Omega_{j} s} \Psi_{i}(L, s) d s=\int_{0}^{t} e^{\Omega_{j} s} \beta_{i}(s) d s .
\end{aligned}
$$

Comparing these global relations to the ones for the massive case on the half-line, many similarities are noted with the addition of two extra terms and the new definitions for the integrals on the left and right boundaries. These similarities ease the algebra necessary to obtain valid expressions for $\Psi_{1}(x, t)$ and $\Psi_{2}(x, t)$.

We now use the global relations and solve for $\hat{\Psi}_{1}(k, t)$ and $\hat{\Psi}_{2}(k, t)$ to apply the inverse Fourier transform. This was accomplished on the half-line by multiplying the global relations by different exponentials and adding and subtracting the respective expressions. Due to the symmetry between the half-line and finite interval cases, the same steps as before can be taken, but new definitions for $C_{1}(k, t)$ and $C_{2}(k, t)$ in (33) and (34) are needed to account for the additional terms left from integrating over the domain. On the finite interval, the new definitions for these expressions are,

$$
\begin{aligned}
\tilde{C}_{j}(k, t)= & (i m) \hat{\Psi}_{1,0}(k)+\left(\Omega_{j}-i k\right) \hat{\Psi}_{2,0}(k) \\
& -(i m) e^{-i k L} B_{L, 1}\left(\Omega_{j}, t\right)+\left(\Omega_{j}-i k\right) e^{-i k L} B_{L, 2}\left(\Omega_{j}, t\right) \\
& +(i m) B_{0,1}\left(\Omega_{j}, t\right)+\left(i k-\Omega_{j}\right) B_{0,2}\left(\Omega_{j}, t\right), \quad j \in\{1,2\}
\end{aligned}
$$

Similar to the half-line, applying the discrete symmetry $k \rightarrow-k$ enables us to eliminate unnecessary boundary terms and obtain the solution on the finite interval. Working through the same argument, we obtain our solutions (41) and (42). We were able to remove the left boundary condition of $\Psi_{2}$, contained in $B_{0,2}$ from (41) and the left boundary condition of $\Psi_{1}$, in $B_{0,1}$ from (42). 


$$
\begin{aligned}
& \Psi_{1}(x, t)=\frac{1}{4 m \pi} \int_{-\infty}^{\infty} \frac{e^{i k x-\Omega_{1} t}}{\Omega_{1}}\left[m \Omega_{1}\left(\hat{\Psi}_{1,0}(k)-\hat{\Psi}_{1,0}(-k)+B_{L, 1}\left(\Omega_{1}, t\right)\left(e^{i k L}-e^{-i k L}\right)\right)\right. \\
& +i m k\left(\hat{\Psi}_{1,0}(k)+\hat{\Psi}_{1,0}(-k)-B_{L, 1}\left(\Omega_{1}, t\right)\left(e^{i k L}+e^{-i k L}\right)\right) \\
& \left.+\left(i \Omega_{1}^{2}+i k^{2}\right)\left(\hat{\Psi}_{2,0}(-k)-\hat{\Psi}_{2,0}(k)+B_{L, 2}\left(\Omega_{1}, t\right)\left(e^{i k L}-e^{-i k L}\right)\right)+(2 i m k) B_{0,1}\left(\Omega_{1}, t\right)\right] d k \\
& +\frac{1}{4 m \pi} \int_{-\infty}^{\infty} \frac{e^{i k x-\Omega_{2} t}}{\Omega_{1}}\left[m \Omega_{1}\left(\hat{\Psi}_{1,0}(k)-\hat{\Psi}_{1,0}(-k)+B_{L, 1}\left(\Omega_{2}, t\right)\left(e^{i k L}-e^{-i k L}\right)\right)\right. \\
& -i m k\left(\hat{\Psi}_{1,0}(k)+\hat{\Psi}_{1,0}(-k)-B_{L, 1}\left(\Omega_{2}, t\right)\left(e^{i k L}+e^{-i k L}\right)\right) \\
& \left.+\left(i \Omega_{1} \Omega_{2}-i k^{2}\right)\left(\hat{\Psi}_{2,0}(-k)-\hat{\Psi}_{2,0}(k)+B_{L, 2}\left(\Omega_{2}, t\right)\left(e^{i k L}-e^{-i k L}\right)\right)-(2 i m k) B_{0,1}\left(\Omega_{2}, t\right)\right] d k \\
& +\frac{1}{2 \pi} \int_{-\infty}^{\infty} e^{i k x} \hat{\Psi}_{1}(-k, t) d k \\
& \Psi_{2}(x, t)=\frac{1}{4 \pi} \int_{-\infty}^{\infty} \frac{e^{i k x-\Omega_{2} t}}{\Omega_{2}}\left[i m\left(\hat{\Psi}_{1,0}(k)-\hat{\Psi}_{1,0}(-k)\right)+\Omega_{2}\left(\hat{\Psi}_{2,0}(k)-\hat{\Psi}_{2,0}(-k)\right)\right. \\
& -i k\left(\hat{\Psi}_{2,0}(k)+\hat{\Psi}_{2,0}(-k)\right)+i m B_{L, 1}\left(\Omega_{2}, t\right)\left(e^{i k L}-e^{-i k L}\right) \\
& \left.+\Omega_{2} B_{L, 2}\left(\Omega_{2}, t\right)\left(e^{-i k L}-e^{i k L}\right)-i k B_{L, 2}\left(\Omega_{2}, t\right)\left(e^{i k L}+e^{-i k L}\right)+(2 i k) B_{0,2}\left(\Omega_{2}, t\right)\right] d k \\
& -\frac{1}{4 \pi} \int_{-\infty}^{\infty} \frac{e^{i k x-\Omega_{1} t}}{\Omega_{2}}\left[i m\left(\hat{\Psi}_{1,0}(k)-\hat{\Psi}_{1,0}(-k)\right)+\Omega_{2}\left(\hat{\Psi}_{2,0}(k)-\hat{\Psi}_{2,0}(-k)\right)\right. \\
& -i k\left(\hat{\Psi}_{2,0}(k)+\hat{\Psi}_{2,0}(-k)\right)+i m B_{L, 1}\left(\Omega_{2}, t\right)\left(e^{i k L}-e^{-i k L}\right) \\
& \left.+\Omega_{2} B_{L, 2}\left(\Omega_{2}, t\right)\left(e^{-i k L}-e^{i k L}\right)-i k B_{L, 2}\left(\Omega_{2}, t\right)\left(e^{i k L}+e^{-i k L}\right)+(2 i k) B_{0,2}\left(\Omega_{2}, t\right)\right] d k k \\
& +\frac{1}{2 \pi} \int_{-\infty}^{\infty} e^{i k x} \hat{\Psi}_{2}(-k, t) d k
\end{aligned}
$$

As before, (41) and (42) are not solutions due to the unknown Fourier transforms, but by the arguments given in Section 2.2 both of the final terms in each equation is zero. Therefore, the solution to the Dirac equation on the finite interval is (41) and (42) with the last terms in each expression removed.

\section{Conclusions}

We have shown the Unified Transform Method is a powerful tool and can be used to obtain closed form solutions to the Dirac equation on the half-line and finite interval. The solutions acquired resemble those in [4] for the Klein-Gordon equation which aligns 
with the interpretation of the Dirac equation as a square root of a diagonal system of Klein-Gordon equations. On the half-line and finite interval, the massless systems held much simpler solutions due to the decoupling of the system of PDEs whereas the coupling in the massive cases provided more involved solutions. Ultimately, this paper gives an example of the utility of the UTM to an important equation from particle physics and showcases the key aspects of this prodigious solution process to partial differential equations.

\section{Acknowledgements}

First, I want to give the utmost thanks to my undergraduate thesis advisor William Green for his guidance and mentorship. In addition, I want to thank Dionyssios Mantzavinos for providing feedback on this problem. The insights of both advanced my undergraduate thesis immensely. Also, I want to thank both of the anonymous referees who provided feedback on this paper.

\section{References}

[1] P.W. Atkins. Quanta: A handbook of concepts. Oxford University Press. (1974), p. 52. ISBN 978-0-19-855493-6.

[2] A. Boutet de Monvel, A. S. Fokas, D. Shepelsky Analysis of the Global Relation for the Nonlinear Schrödinger Equation on the Half-line, Lett. Math. Phys. 653 (2003), 199-212.

[3] R.C. Cascaval, C.T. Hunter, Linear and nonlinear Schrödinger equations on simple networks, Libertas Math (2010).

[4] B. Deconinck, Q. Guo, E. Shilzerman, and V. Vasan, Fokas's Unified Transform Method for Linear Systems, Quart. Appl. Math. 76 (2018), 463-488 .

[5] B. Deconinck, B. Pelloni, N. Sheils Non-steady-state heat conduction in composite walls, Proc. R. Soc. Lond. Ser. A Math. Phys. Eng. Sci. 4702165 (2014), 20130605, http://arxiv.org/abs/1402.3007

[6] B. Deconinck, N. Sheils Interface problems for dispersive equations, (2014), http://arxiv.org/abs/1405.3307 submitted for review .

[7] B. Deconinck, T. Trogdon, and V. Vasan, The method of Fokas for solving linear partial differential equations, SIAM Rev. 56 (2014), no 1, 159-186, DOI $10.1137 / 110821871$.

[8] The Editors of Encyclopedia Britannica. Positron https://www.britannica.com/science/positron (updated June 03, 2013). 
[9] N. Flyer and A.S. Fokas, A hybrid analytical-numerical method for solving evolution partial differential equations. I. the half-line, Proc. R. Soc. Lond. Ser. A Math. Phys. Eng. Sci., 464 (2008), pp. 1823-1849.

[10] A. S. Fokas A unified transform method for solving linear and certain nonlinear PDEs, Proc. R. Soc. Lond. Ser. A Math. Phys. Eng. Sci. 453 (1997), 1411-1443.

[11] A.S. Fokas, A Unified Approach to Boundary Value Problems, CBMS-NSF Regional Conf. Ser. in Appl. Math. 78, SIAM, Philadelphia, PA, 2008.

[12] A. S. Fokas, I. M. Gel'fand Integrability of Linear and Nonlinear Evolution Equations and the Associated Nonlinear Fourier Transforms, Lett. Math. Phys. 32 (1994), 189-210.

[13] A. S. Fokas, A. R. Its The Linearization of the Initial-Boundary Value Problem of the Nonlinear Schrödinger Equation, SIAM J. Appl. Math. 273 (1996), 738-764.

[14] A.S. Fokas, B. Pelloni, Boundary value problems for Boussinesq type systems, Math. Phys. Anal. Geom. 8 (2005), no. 1, 59-96, DOI 10.1007/s11040-004-1650-6. MR2136653

[15] A.S. Fokas, B. Pelloni, A transform method for linear evolution PDEs on a finite interval, IMA J. Appl. Math (2005).

[16] E.-N.G. Grylonakis, C.K. Filelis-Papadopoulos, G.A. Gravvanis, A.S. Fokas. An iterative spatial-stepping numerical method for linear elliptic PDEs using the Unified Transform. Journal of Computational and Applied Mathematics 352, pages 194-209.

[17] N. Sheils, B. Deconinck, Interface problems for dispersive equations, Stud. in Appl. Math (2015).

[18] N. Sheils, B. Deconinck, The time-independent Schrödinger equation with piecewise constant potentials, EJAM (2018).

[19] A.G. Sifalakis, A.S. Fokas, S.R. Fulton, Y.G. Saridakis. The generalize DirichletNeumann map for linear elliptic PDEs and its numerical implementation. Journal of Computational and Applied Mathematics 219, pages 9-34.

[20] D.A. Smith, Unified Transform Method for boundary value problems https://unifiedmethod.azurewebsites.net/applications.html

[21] B. Thaller, The Dirac equation. Texts and Monographs in Physics. Springer-Verlag, Berlin, 1992.

[22] T.Hey, P.Walters (2009). The New Quantum Universe. Cambridge University Press. p. 228. ISBN 978-0-521-56457-1. 